九州大学学術情報リポジトリ

Kyushu University Institutional Repository

\title{
STRONG CONVERGENCE OF AN UNBOUNDED STOCHASTIC APPROXIMATION ALGORITHM UNDER GENERAL CONDITIONS
}

Watanabe, Masafumi

Department of Applied Mathematics, Fukuoka University

https://doi.org/10.5109/13426

出版情報: Bulletin of informatics and cybernetics. 25 (1/2), pp.109-123，1992-03. Research Association of Statistical Sciences

バージョン :

権利関係 : 


\title{
STRONG CONVERGENCE OF AN UNBOUNDED STOCHASTIC APPROXIMATION ALGORITHM UNDER GENERAL CONDITIONS
}

\author{
By
}

Masafumi Watanabe*

\begin{abstract}
Under general conditions on the observation processes the almost sure convergence properties of an unbounded and nonlinear Robbins-Monro type stochastic approximation algorithm are investigated.
\end{abstract}

\section{Introduction}

In the classical studies of stochastic approximation, essentially it was assumed that the observations are independent. But many practical applications involve the use of correlated data. Consequently classical stochastic approximation convergence results are often not applicable. Recently, several efforts have been made to get rid of it, for example it can be replaced by mixing conditions or ergodic conditions. Borodin [1], Fritz [3], Györfi [3], Kushner and Clark [4], Ljung [5, 6] investigated the case when the observations may not be independent. For linear regression Fritz [2] and Györfi [3] formulate a completely deterministic problem, by which the strong consistency can be deduced if for the observations the strong law of large numbers is applied, therefore the conditions of strong consistency of linear stochastic approximation are as general as the conditions of the strong law of large numbers. Thus the effects of the randomness and the iteration were separated. By the similar motivation and for nonlinear regression Ljung [6] and Kushner and Clark [4] have obtained interesting results under the assumption that the algorithms are bounded.

Generally the bounded condition is not easily established for the family of stochastic approximation algorithms. In this paper we shall investigate the strong convergence of an umbounded and nonlinear Robbins-Monro type stochastic approximation algorithm under general conditions on the observations.

\section{The Robins-Monro Stochastic Approximation Algorithm}

Throughout the paper $H$ denotes a real separable Hilbert space with an inner product $\langle\cdot, \cdot\rangle$ and a norm $\|\cdot\|$, and $O$ denotes the null element of $H$. Let $(\Omega, \mathscr{A}, P)$ be a probability space.

\footnotetext{
* Department of Applied Mathematics, Fukuoka University Fukuoka 814-01. Japan
} 
Let $M(x)$ be a Borel measurable transformation from $H$ into itself. For each $n \geq 1$, let a function $Y_{n}(x, \omega)$ which takes value in $H$ and is defined for $(x, \omega) \in H \times \Omega$ be measurable with respect to $\mathbb{B} \times \mathcal{A}$, where $\mathscr{B}$ is the Borel field of $H$. Then we consider the following Robbins-Monro stochastic approximation algorithm for finding the solution of the equation

$$
M(x)=O
$$

Algorithm:

$$
X_{n+1}=X_{n}-a_{n} Y_{n}, \quad n=1,2, \ldots
$$

where $X_{1}$ is a constant element in $H, Y_{n}=Y_{n}\left(X_{n}(\omega), \omega\right), \omega \in \Omega$ and $\left\{a_{n}\right\}$ is a sequence of positive numbers. Throughout the paper let us put

$$
Z_{n}(x, \omega)=Y_{n}(x, \omega)-M(x), n=1,2, \ldots,(x, \omega) \in H \times \Omega .
$$

Then (2.2) can be rewritten the following

$$
X_{n+1}=X_{n}-a_{n}\left\{M\left(X_{n}\right)+Z_{n}\right\}, \quad n=1,2, \ldots,
$$

where

$$
Z_{n}=Z_{n}\left(X_{n}(\omega),(\omega), \quad \omega \in \Omega\right. \text {. }
$$

Fritz [2] and Györfi [3] have investigated the above problem under the conditions that $M(x)=A x+b$ and $Y_{n}(x, \omega)=A_{n}(\omega) x+b_{n}(\omega)$, where $A, A_{n}$ are bounded linear operators and $b, b_{n}$ are elements in $H$. And the a.s. convergence of process (2.2) has been discussed under the assumptions that $A_{n}^{\prime}$ s and $b_{n}^{\prime}$ s arithmetic means converge to $A$ and $b$ respectively. Kushner and Clark [4] and Ljung [6] have investigated more general case and have given a.s. convergence results. In [4] and [6], they assumed that

$$
\sup _{n}\left\|X_{n}\right\|<\infty \quad \text { a.s. . }
$$

Condition (2.5) appears to be a reasonable condition but is not easily established for the family of algorithms represented by (2.2). In this paper we note that condition (2.5) will not be assumed.

\section{Preliminaries}

In this section we shall give two lemmas which will be needed the proof of the theorem in the section 5 (Theorem B).

Lemma 1. Let $\left\{a_{n}\right\}$ and $\left\{v_{n}\right\}$ be sequences of real numbers such that

$$
\begin{gathered}
a_{n}>0, n=1,2, \ldots, \quad \lim _{n \rightarrow \infty} a_{n}=0, \\
\sum_{n=1}^{x} a_{n}=x, \quad \sup _{n}\left|a_{n}^{-1}-a_{n+1}^{-1}\right|<\infty,
\end{gathered}
$$

$$
v_{n} \geq 0, n=1,2, \ldots, \quad \sum_{n=1}^{\infty} v_{n}<\infty .
$$


And let $\left\{x_{n}\right\}$ be a sequence of nonnegative numbers and $\left\{w_{n}\right\}$ be a sequence of real numbers which satisfy the following enequality

(iii) $\quad x_{n+1} \leq\left(1-a_{n}+v_{n}\right) x_{n}+w_{n}, n=1,2, \ldots$

Suppose that let $T$ be any positive number then there exists a positive number $W_{T}$ such that

$$
\sup _{n} \max _{n \leqslant k<m(n, T)}\left|\sum_{i=n}^{k} w_{i}\right| \leq W_{T}
$$

where

$$
m(n, T)=\max \left\{k \mid \sum_{i=n}^{k} a_{i} \leq T\right\}
$$

Then it follows that there exist two positive numbers $T_{0}$ and $W_{0}$ such that

$$
\sup _{n} x_{n} \leq\left(W_{T_{0}}+1\right) W_{0} \text {. }
$$

Proof. It follows from (i) that there exists a positive integer $N$ such that

$$
\begin{aligned}
\left(1-a_{i}+v_{i}\right) & =\left(1-a_{i}\right)\left\{1+\left(1-a_{i}\right)^{-1} v_{i}\right\} \\
& \leq\left(1-a_{i}\right)\left(1+2 v_{i}\right) \quad \text { for all } i \geq N .
\end{aligned}
$$

Let us put

$$
A(m, n)=\left\{\begin{array}{cl}
\prod_{i=m}^{n}\left(1-a_{i}\right)\left(1+2 v_{i}\right) & \text { if } n \leq m \\
1 & \text { if } n=m-1 .
\end{array}\right.
$$

Condition (ii) yields that there exists a positive number $\bar{v}$ such that

$$
1 \leq \prod_{i=m}^{\infty}\left(1+2 v_{i}\right) \leq \bar{v} \quad \text { for all } m \geq 1 \text {. }
$$

Hence, by (3.5) and (3.6) we have

$$
A(m, n) \leq \bar{v} \exp \left(-\sum_{i=m}^{n} a_{i}\right) \quad \text { for } N \leq m \leq n .
$$

Let us define $T_{0}>0$ by

$$
\begin{gathered}
a_{n} \leq T_{0} / 4 \quad n=1,2, \ldots \\
\bar{v} / \exp \left(T_{0} / 2\right) \leq \frac{1}{2} .
\end{gathered}
$$

Then, by (3.2) we have

$$
\sum_{i=n}^{m\left(n, T_{1}\right)-1} a_{i} \leq T_{0} \quad, n \geq N
$$


and

$$
\sum_{i=n}^{m\left(n, T_{1}\right)+1} a_{i}>T_{0} \quad, n \geq N
$$

Further, (3.8) and (3.11) imply

$$
\sum_{i=n}^{m\left(n, T_{0}\right)-1} a_{i}>T_{0}-a_{m}\left(n, T_{0}\right)-a_{m\left(n, T_{1}\right)-1} \geq \frac{T_{0}}{2} \quad, n \geq N .
$$

Hence by (3.10) and (3.12) we obtain

$$
\frac{T_{0}}{2} \sum_{i=n}^{m\left(n, T_{0}\right)-1} a_{i} \leq T_{0}, \quad, n \geq N
$$

And we also have from (3.9) and (3.13) that

$$
A\left(n, m\left(n, T_{0}\right)-1\right) \leq \frac{1}{2} \quad, n \geq N .
$$

If (3.1) holds then we can easily shown from (i) and (ii) that

$$
\sup _{n \geq N} \max _{n \leq k<m\left(n, T_{1}\right)}\left|\sum_{i=n}^{k} w_{i} A(i+1, k)\right| \leq\left(1+\bar{v} \bar{A}_{N}\right) W_{T_{n}},
$$

where

$$
\bar{A}_{N}=\sup _{n \geq N}\left\{\sum_{i=N}^{n} a_{i} \prod_{j=i+1}^{n}\left(1-a_{j}\right)\right\}+2 \sum_{n=1}^{\infty} v_{n} .
$$

We note that (i) yields $\bar{A}_{N}<x$.

Next we shall define a subsequence $\left\{x_{n_{r}}\right\} \subset\left\{x_{n}\right\}$ by the following way.

Let $n_{0}=N$ and $n_{r}=m\left(n_{r-1}, T_{0}\right), r=1,2, \ldots$

Then we have from (iii), (3.14) and (3.15) that

$$
\begin{aligned}
x_{n 1} & \leq A\left(n_{0}, n_{1}-1\right) x_{n_{1}}+\left|\sum_{i=n_{0}}^{n_{1}-1} w_{i} A\left(i+1, n_{1}-1\right)\right| \\
& \leq x_{N}+2\left(1+\bar{v} \bar{A}_{N}\right) W_{T_{i n}}, \\
x_{n_{2}} & \leq \frac{1}{2} x_{n_{1}}+\left(1+\bar{v} \bar{A}_{N}\right) W_{T_{i 1}} \\
& \leq x_{N}+2\left(1+\bar{v} \bar{A}_{N}\right) W_{T_{i 1}} .
\end{aligned}
$$

Hence we have

$$
\sup _{r} x_{n_{r}} \leq x_{N}+2\left(1+\bar{v} \bar{A}_{N}\right) W_{T_{1}}
$$

Let $n$ be any integer such that $n \geq N$. Then there exists positive integer $r$ such that

$$
n_{r} \leq n<n_{r+1} \text {. }
$$

Hence we have from (iii), (3.7) and (3.16) that 


$$
\begin{aligned}
x_{n} & \leq A\left(n_{r}, n-1\right) x_{n_{r}}+\max _{n_{,} \leq k<n_{r+1}}\left|\sum_{i=n_{r}}^{k} w_{i} A(i+1, k)\right| \\
& \leq \bar{v} x_{n_{r}}+\left(1+\bar{v} \bar{A}_{N}\right) W_{T_{0}} \\
& \leq 3 \bar{v}\left(1+\bar{v} \bar{A}_{N}\right)\left(x_{N}+W_{T_{0}}\right), \quad n_{r} \leq n<n_{r+1} .
\end{aligned}
$$

Hence we have

$$
\sup _{n} x_{n} \leq\left(1+W_{T_{0}}\right) W_{0},
$$

where $W_{0}=\max \left\{3 \bar{v}\left(1+\bar{v} \bar{A}_{N}\right), 3 \bar{v}\left(1+\bar{v} \bar{A}_{N}\right) \bar{x}_{N}\right\}$ and $\bar{x}_{N}=\max \left\{x_{1}, x_{2}, \ldots, x_{N}\right\}$.

Q.E.D.

Lemma 2. Suppose that the conditions of Lemma 1 hold but with the following condition replacing (3.1),

for any positive number $T$ it follows that

$$
\lim _{n \rightarrow \infty} \max _{n \leq k<m(n, T)}\left|\sum_{i=n}^{k} w_{i}\right|=0 .
$$

Then it holds that

$$
\lim _{n \rightarrow \infty} x_{n}=0 .
$$

Proof. It follows from Lemma 1 that there exists positive numbers $W_{1}$ such that

$$
\sup _{n} x_{n} \leq W_{1},
$$

where $W_{1}=\left(1+W_{T_{0}}\right) W_{0}$.

Let $N$ be a positive integer which is given in the proof of Lemma 1 . Let $\varepsilon$ be any real number such that

$$
0<\varepsilon<\frac{1}{2}
$$

And let us define a positive number $T_{1}$ by the following

$$
\begin{gathered}
2 \leq T_{1} \\
\bar{v} / \exp \left(T_{1} / 2\right)<\varepsilon,
\end{gathered}
$$

where $\bar{v}$ is also given in the proof of Lemma 1 .

By the same arguments of (3.14) and from (3.20) we have

$$
A\left(n, m\left(n, T_{1}\right)-1\right) \leq \bar{v} \exp \left(-T_{1} / 2\right)<\varepsilon<1 / 2, n \geq N .
$$

And (3.17) implies that there exists a positive integer $N_{1}=N_{1}\left(\varepsilon, T_{1}\right) \geq N$ such that

$$
\sup _{n \geq N_{1}} \max _{n \leq k<m\left(n, T_{1}\right)}\left|\sum_{i=n}^{k} w_{i}\right|<\varepsilon .
$$


Hence, by the same arguments of the proof of (3.15) we have

$$
\sup _{n \geq N_{1}} \max _{n \leq k<m\left(n, T_{1}\right)}\left|\sum_{i=n}^{k} w_{i} A(i+1, k)\right| \leq \varepsilon\left(1+\bar{v} \bar{A}_{N_{1}}\right) .
$$

Next we shall define a subsequence $\left\{x_{n}\right\} \subset\left\{x_{n}\right\}$ by the following way. Let us take $n_{0}=$ $N_{1}$ and $n_{r}=m\left(n_{r-1}, T_{1}\right), r=1,2, \ldots$ Since $N \leq N_{1}$ and $\sup _{n} x_{n} \leq W_{1}$ we have from (3.21) and (3.22)

$$
\begin{gathered}
x_{n_{1}} \leq \varepsilon\left(W_{1}+1+\bar{v} \bar{A}_{N_{1}}\right)<2 \varepsilon\left(W_{1}+1+\bar{v} \bar{A}_{N_{1}}\right) . \\
x_{n_{2}} \leq \frac{1}{2} x_{n_{1}}+\varepsilon\left(W_{1}+1+\bar{v} \bar{A}_{N_{1}}\right)<2 \varepsilon\left(W_{1}+1+\bar{v} \bar{A}_{N_{1}}\right) .
\end{gathered}
$$

Hence we obtain

$$
\sup _{r} x_{n_{\mathrm{r}}} \leq 2 \varepsilon\left(1+W_{1}+\bar{v} \bar{A}_{N_{1}}\right)
$$

For any $n \geq n_{1}=m\left(N_{1}, T_{1}\right)$ there exists a positive integer $r$ such that $n_{r} \leq n<n_{r+1} 1$ Then it follows from (iii), (3.22) and (3.23) that

$$
\begin{aligned}
x_{n} & \leq A\left(n_{r}, n\right) x_{n_{r}}+\max _{n_{r} \leq k<n_{r+1}}\left|\sum_{i=n}^{k} w_{i} A(i+1, k)\right| \\
& \leq \bar{v} x_{n_{r}}+2 \varepsilon\left(1+W_{1}+\bar{v} \bar{A}_{N_{1}}\right) \\
& \leq 3 \varepsilon \bar{v}\left(1+W_{1}+\bar{v} \bar{X}_{N_{1}}\right) .
\end{aligned}
$$

Hence we obtain

$$
\varlimsup_{n \rightarrow \infty} x_{n} \leq 3 \varepsilon \bar{v}\left(1+W_{1}+\bar{v} \bar{A}_{N_{1}}\right) .
$$

Q.E.D.

Let $\left\{a_{n}\right\}$ and $\left\{w_{n}\right\}$ be sequences of real numbers which are given in Lemma 1 . And we suppose that

$$
a_{n}>a_{n+1} \quad, n=1,2, \ldots
$$

and

$$
w_{n}=a_{n} y_{n} \quad, n=1,2, \ldots
$$

Then we shall consider the following four conditions.
I. $\sum_{n=1}^{\infty} a_{n} y_{n} \quad$ converges,
II. $\lim _{n \rightarrow \infty} a_{n} \sum_{i=1}^{n} y_{i}=0$,
III. $\lim _{n \rightarrow \infty} \sum_{i=1}^{n} a_{i} \prod_{j=i+1}^{n}\left(1-a_{j}\right) y_{i}=0$, 
IV. $\lim _{n \rightarrow \infty} \max _{n \leq k<m(n . T)}\left|\sum_{i=n}^{k} a_{i} y_{i}\right|=0$,

where $m(n, T)$ is defined by (3.2).

Since $a_{n} \downarrow 0$ as $n \rightarrow \infty$ and by using Kronecker's Lemma we have

$$
\mathrm{I} \Rightarrow \mathrm{II} \text {. }
$$

Noting $\sup _{n}\left|a_{n}^{-1}-a_{n+1}^{-1}\right|<x$ we have

$$
\text { II } \Rightarrow \text { III. }
$$

And we can also show that

$$
\text { III } \Rightarrow \text { IV }
$$

Hence it follows that

$$
\mathrm{I} \Rightarrow \mathrm{II} \Rightarrow \mathrm{III} \Rightarrow \mathrm{IV}
$$

Next we suppose that

$$
a_{n}=n^{-1}, \quad n=1,2, \ldots
$$

Then we can show that

$$
(n-2) e^{T} \leq m(n, T) \leq n e^{T}, \quad n \geq 2
$$

Hence we can prove that

$$
\text { IV } \Rightarrow \text { II. }
$$

And we can also prove that

$$
\text { II } \Leftrightarrow \text { III. }
$$

In this case it follows that

$$
\text { II } \Leftrightarrow \text { III } \Leftrightarrow \text { IV. }
$$

Let us suppose that

$$
a_{n}=n^{-a}, \quad y_{n}=n^{-\beta}, \quad n=1,2, \ldots,
$$

where $0<\alpha, \beta$ and $\alpha+\beta=1$. In this case it is easily seen that I and II do not hold. And we have

$$
m(n, T) \sim\left\{n^{1-\alpha}+(1-\alpha) T\right\}^{(1-\alpha)^{-1}} .
$$

Hence it follows that

$$
\sum_{i=n}^{m(n, T)} a_{i} y_{i}=\sum_{i=n}^{m(n, T)} i^{-1} \sim(1-\alpha)^{-1} \log \left\{1+(1-\alpha) T n^{\alpha-1}\right\} .
$$

Hence IV holds. 
Thus condition IV can be considered more general than the other conditions I to III.

\section{Assumptions}

Let $\theta \in H$ be the solution of the equation (2.1) and let $M(x),\left\{a_{n}\right\},\left\{X_{n}\right\},\left\{Y_{n}\right\}$ and $\left\{Z_{n}\right\}$ be given in the section 1 .

Moreover let $\Omega_{0}$ be a subset of $\Omega$ such that

$$
\Omega_{0} \in \mathscr{A} \text { and } P\left(\Omega_{0}\right)=1 .
$$

We make the following assumptions.

A0: There exists a sequence of positive random variables $\left\{\delta_{n}(\omega)\right\}$ such that

(i) $\delta_{n}(\omega) \geq \delta_{n+1}(\omega), n=1,2, \ldots$ and $\lim \delta_{n}(\omega)=0, \quad \omega \in \Omega_{0}$,

(ii) $\sup _{n} \delta_{n}(\omega) \delta_{n+1}^{-1}(\omega)<\infty, \quad \omega \in \Omega_{0}$,

(iii) $\sup _{n} \delta_{n}(\omega)\left\|X_{n}(\omega)\right\|<\infty, \omega \in \Omega_{0}$.

A1: $\left\{a_{n}\right\}$ is a decreasing sequence of positive numbers.

(i) $\sum_{n=1}^{\infty} a_{n}^{2}<x, \sum_{n=1}^{\infty} a_{n}=x$,

(ii) $\sup _{n}\left|a_{n}^{-1}-a_{n+1}^{-1}\right|<x$.

A2: There exists a positive constant $C$ such that

$$
\|M(x)\| \leq C(\|x\|+1), x \in H .
$$

A3: $\inf _{\varepsilon<\|x-\theta\|<\varepsilon^{-1}}\langle M(x), x-\theta\rangle>0$ for any $0<\varepsilon<1$.

A4: There exists a sequence of nonnegative random variables $\left\{\alpha_{n}(\omega)\right\}$ such that

(i) $\left\|Z_{n}(x, \omega)\right\| \leq \alpha_{n}(\omega)(\|x\|+1), n=1,2, \ldots,(x, \omega) \in H \times \Omega_{0}$,

(ii) $\sum_{n=1}^{\infty} a_{n}^{2} \alpha_{n}^{2}(\omega)<\infty, \omega \in \Omega_{0}$.

A5: Let $\left\{\delta_{n}(\omega)\right\}$ be given in A0. And there exists a nonnegative random variables $\beta_{0}(\omega)$ such that

(i) $\sup _{n} a_{n} \delta_{n}^{-3}(\omega)\left\|\sum_{i=1}^{n} Z_{i}(x, \omega)\right\| \leq \beta_{0}(\omega)(\|x\|+1),(x, \omega) \in H \times \Omega_{0}$,

(ii) $\sup _{n} a_{n} \delta_{n}^{-3}(\omega)\left\|\sum_{i=1}^{n}\left\{Z_{i}(x, \omega)-Z_{i}(y, \omega)\right\}\right\| \leq \beta_{0}(\omega)\|x-y\|$,

$(x, \omega),(y, \omega) \in H \times \Omega_{0}$. 
A6:

(i) $\sum_{n=1}^{\infty} a_{n} \delta_{n}(\omega)<x, \omega \in \Omega_{0}$,

(ii) $\sum_{n=1}^{\infty} a_{n} \delta_{n}(\omega) \alpha_{n}(\omega)<x, \omega \in \Omega_{()}$

Assumptions A0 to A6 will be used in Theorem A which will be given in the next section. And the following assumptions B0 to B6 will be also used in Theorem B.

Let $m(n, T)$ be defied by (3.2).

B0: Assumption A0 holds.

B1: Assumption A1 holds.

B2: Assumption A2 holds.

B3: There exists a positive number $\lambda$ such that

$$
\langle M(x), x-\theta\rangle \geq \lambda\|x-\theta\|^{2}, x \in H .
$$

B4: Assumption A4 holds.

B5: For any positive number $T$ there exists a positive random variable $\beta_{T}(\omega)$ such that

(i) $\sup _{n} \max _{n \leq k<m(n, T)}\left\|\sum_{i=n}^{k} a_{i} \delta_{i}^{-2}(\omega) Z_{i}(x, \omega)\right\| \leq \beta_{T}(\omega)(\|x\|+1),(x, \omega) \in H \times \Omega_{0}$,

(ii) $\sup _{n} \max _{n \leq k<m(n, T)}\left\|\sum_{i=n}^{k} a_{i} \delta_{i}^{-2}(\omega)\left\{Z_{i}(x, \omega)-Z_{i}(y, \omega)\right\}\right\|$

$$
\leq \beta_{T}(\omega)\|x-y\|,(x, \omega),(y, \omega) \in H \times \Omega_{0},
$$

B6: $\sup _{n} \sum_{i=n}^{m(n, T)} a_{i} \alpha_{i}(\omega)<x,(x, \omega) \in H \times \Omega_{0}$.

REMARK. Let us assume that

$\mathrm{A}^{\prime} 0$ : there exist a sequence of nonnegative random variables $\left\{\gamma_{n}(\omega)\right\}$ and a sequence of positive random variables $\left\{\delta_{n}(\omega)\right\}$ such that

(i) A0(i), (ii) hold

(ii) $\left\langle\theta-x, Y_{n}(x, \omega)\right\rangle \leq \gamma_{n}(\omega)(\|x\|+1)$, $n=1,2, \ldots,(x, \omega) \in H \times \Omega_{0}$,

(iii) $\sum_{n=1}^{\infty} a_{n} \delta_{n}(\omega) \gamma_{n}(\omega)<\infty, \omega \in \Omega_{0}$.

Then it follows from Lemma $1([9])$ that A0(iii) holds. 


\section{Results}

In this section we give two theorems (Theorems A and $\mathrm{B}$ ) which are the main results of this paper.

Theorem A. Assume A0 to A6. Then it holds that

$$
\lim _{n \rightarrow \infty}\left\|X_{n}-\theta\right\|=0 \quad \text { a.s. . }
$$

Proof. Without loss of generality we can assume $\theta=\boldsymbol{O}$. Here and latter, we take $\theta=\boldsymbol{O}$. and $K$ denotes positive random variables which are not necessarily the same.

By virture of the algorithm (2.2) we obtain

$$
\left\|X_{n+1}\right\|^{2}=\left\|X_{n}\right\|^{2}-2 a_{n}\left\langle X_{n} . M\left(X_{n}\right)+Z_{n}\right\rangle+a_{n}^{2}\left\|M\left(X_{n}\right)+Z_{n}\right\|^{2} .
$$

Substituting A2 and A4(i) into (5.2) gives

$$
\left\|X_{n+1}\right\|^{2} \leq\left(1+K a_{n}^{2}+K a_{n}^{2} \alpha_{n}^{2}\right)\left\|X_{n}\right\|^{2}-2 a_{n}\left\langle X_{n}, M\left(X_{n}\right)+Z_{n}\right\rangle+K a_{n}^{2}\left(\alpha_{n}^{2}+1\right) .
$$

First we shall show that

$$
\sum_{n=1}^{\infty} a_{n}\left\langle X_{n}, Z_{n}\right\rangle \quad \text { converges a.s. . }
$$

Define $S_{n}(x)=a_{n} \sum_{i=1}^{n} Z_{i}(x), \quad S_{0}(x)=O$ and

$$
W(m, n)=\sum_{i=m}^{n} a_{i}\left\langle X_{i}, Z_{i}\right\rangle, \quad n \leq m .
$$

Since $Z_{i}(x)=a_{i}^{-1} S_{i}(x)-a_{i-1}^{-1} S_{i-1}(x), W(m, n)$ can be rewritten the following formula

$$
\begin{aligned}
W(m, n) & =\sum_{i=m}^{n}\left\langle X_{i}, S_{i}\left(X_{i}\right)-a_{i} a_{i-1}^{-1} S_{i-1}\left(X_{i}\right)\right\rangle \\
& =W_{1}(m, n)+W_{2}(m, n)+W_{3}(m, n), m \leq n,
\end{aligned}
$$

where

$$
\begin{gathered}
\mathrm{W}_{1}(m, n)=\sum_{i=m}^{n}\left\langle X_{i}, S_{i}\left(X_{i}\right)-S_{i-1}\left(X_{i}\right)\right\rangle, \\
W_{2}(m, n)=\sum_{i=m}^{n}\left\langle X_{i},\left(1-a_{i} a_{i-1}^{-1}\right) S_{i-1}\left(X_{i-1}\right)\right\rangle
\end{gathered}
$$

and

$$
W_{3}(m, n)=\sum_{i=m}^{n}\left\langle X_{i}, a_{i} a_{i-1}^{-1}\left\{S_{i-1}\left(X_{i-1}\right)-S_{i-1}\left(X_{i}\right)\right\}\right\rangle .
$$

Then we have 


$$
\begin{aligned}
\left|W_{1}(m, n)\right| & \leq\left|\sum_{i=m}^{n-1}\left\langle X_{i}-X_{i+1}, S_{i}\left(X_{i}\right)\right\rangle\right|+\left|\left\langle X_{m}, S_{m-1}\left(X_{m-1}\right)\right\rangle\right|+\left|\left\langle X_{n}, S_{n}\left(X_{n}\right)\right\rangle\right| \\
& \leq \sum_{i=m}^{n}\left\|S_{i}\left(X_{i}\right)\right\| \cdot\left\|X_{i}-X_{i+1}\right\|+\left\|X_{m}\right\| \cdot\left\|S_{m-1}\left(X_{m-1}\right)\right\|+\left\|X_{n}\right\| \cdot\left\|S_{n}\left(X_{n}\right)\right\| .
\end{aligned}
$$

Since $X_{i+1}=X_{i}-a_{i} Y_{i}$, it follows from A5 that

$$
\begin{aligned}
\left|W_{1}(m, n)\right| \leq & \sum_{i=m}^{n} \beta_{0} a_{i} \delta_{i}^{3}\left(\left\|X_{i}\right\|+1\right)\left\|Y_{i}\right\|+\beta_{0} \delta_{m-1}^{3}\left(\left\|X_{m-1}\right\|+1\right)\left\|X_{m}\right\| \\
& +\beta_{0} \delta_{n}^{3}\left(\left\|X_{n}\right\|+1\right)\left\|X_{n}\right\| .
\end{aligned}
$$

A2 and A4(i) imply

$$
\left\|Y_{i}\right\| \leq K \alpha_{i}\left(\left\|X_{i}\right\|+1\right), i=1,2 \ldots .
$$

Hence it follows from (5.5) and A0 that

$$
\left|W_{1}(m, n)\right| \leq K\left(\sum_{i=m}^{n} a_{i} \delta_{i} \alpha_{i}+\delta_{m-1}+\delta_{n}\right) .
$$

Hence by A0(i) and A6(ii) we obtain

$$
\lim _{m . n \rightarrow \infty}\left|W_{1}(m, n)\right|=0 \quad \text { on } \Omega_{1} .
$$

Since $\left\{a_{n}\right\}$ is decreasing and $1-a_{i} a_{i-1}^{-1}=a_{i}\left(a_{i}^{-1}-a_{i-1}^{-1}\right)$, it follows from A0 and A1(ii) that

$$
\left|W_{2}(m, n)\right| \leq K \sum_{i=m}^{n} a_{i-1} \delta_{i-1}
$$

Hence A6(i) implies

$$
\lim _{m . n \rightarrow \infty}\left|W_{2}(m, n)\right|=0 \quad \text { on } \Omega_{0} .
$$

It follows from $\mathrm{A} 5$ (ii) that

$$
\left|W_{3}(m, n)\right| \leq \sum_{i=m}^{n} \beta_{0} a_{i} \delta_{i-1}^{3}\left\|X_{i}\right\|\left\|Y_{i-1}\right\| .
$$

Hence by $\mathrm{A} 0$ and (5.5) we obtain

$$
\left|W_{3}(m, n)\right| \leq K \sum_{i=m}^{n} a_{i-1} \delta_{i-1} \alpha_{i-1}
$$

Hence A6 (ii) implies

$$
\lim _{m, n \rightarrow \infty}\left|W_{3}(m, n)\right|=0 \quad \text { on } \Omega_{(1)}
$$

Thus (5.4) has been proved. 
Next we shall show (5.1). Define $U_{n}=2 a_{n}\left\langle X_{n}, M\left(X_{n}\right)\right\rangle, V_{n}=K a_{n}^{2}\left(\alpha_{n}^{2}+1\right)$ and $W_{n}=2 a_{n}\left\langle X_{n}, Z_{n}\right\rangle$. Then (5.3) yields

$$
\left\|X_{n+1}\right\|^{2} \leq\left(1+K V_{n}\right)\left\|X_{n}\right\|^{2}-U_{n}+K V_{n}-W_{n} .
$$

Noting $U_{n} \geq 0, \sum_{n=1}^{\infty} V_{n}<x$ a.s., $\sum_{n=1}^{\infty} W_{n}$ converges a.s. and using the following inequality

$$
\begin{aligned}
0 \leq\left\|X_{n+1}\right\|^{2} \leq & \left\|X_{1}\right\|^{2} \prod_{i=1}^{n}\left(1+K V_{i}\right)-\sum_{i=1}^{n} U_{i} \prod_{j=i+1}^{n}\left(1+K V_{i}\right) \\
& +\sum_{i=1}^{n}\left(K V_{i}-W_{i}\right) \prod_{j=i+1}^{n}\left(1+K V_{i}\right),
\end{aligned}
$$

we can obtain

$$
\sup _{n}\left\|X_{n}\right\|<x \quad \text { a.s. },
$$

and

$$
\sum_{n=1}^{\infty} a_{n}\left\langle X_{n}, M\left(X_{n}\right)\right\rangle<\propto \quad \text { a.s. }
$$

Hence it follows from A1(i) and A3 that there exists a subsequence $\left\{X_{n_{h}}\right\} \subset\left\{X_{n}\right\}$ such that

$$
\lim _{k \rightarrow \infty}\left\|X_{n_{k}}\right\|=0 \quad \text { a.s. }
$$

Moreover (5.2) yields that

$$
\left|\left\|X_{n}\right\|^{2}-\left\|X_{m}\right\|^{2}\right| \leq \sum_{i=m}^{n-1} a_{i}^{2}\left\|Y_{i}\right\|^{2}+\sum_{i=n}^{n-1} U_{i}+\left|\sum_{i=m}^{n-1} W_{i}\right| .
$$

We note that (5.5), A1(i) and A4(ii) imply

$$
\sum_{n=1}^{\infty} a_{n}^{2}\left\|Y_{n}\right\|^{2}<\infty \quad \text { a.s. . }
$$

Hence (5.4), (5.7) and (5.8) imply

$$
\lim _{m, n \rightarrow \infty}\left|\left\|X_{n}\right\|^{2}-\left\|X_{m}\right\|^{2}\right|=0 \quad \text { a.s. . }
$$

Hence (5.1) has been proved.

Q.E.D.

Theorem B. Assume B0 to B6. Then (5.1) holds.

Proof. Substituting B3 into (5.2) we obtain

$$
\left\|X_{n+1}\right\|^{2} \leq\left(1-2 \lambda_{n} a_{n}+K a_{n}^{2}+K a_{n}^{2} a_{n}^{2}\right)\left\|X_{n}\right\|^{2}-2 a_{n}\left\langle X_{n}, Z_{n}\right\rangle+K a_{n}^{2}\left(\alpha_{n}^{2}+1\right) .
$$


First we note that B1 and B4(ii) yield that

$$
\limsup _{n \rightarrow \infty} \sum_{i=n}^{m(n, T)} a_{i}^{2}\left(\alpha_{i}^{2}+1\right)=0 \quad \text { on } \Omega_{0}
$$

for any $T>0$. Next we shall show that, for any $T>0$ there exists a positive random variables $W_{0}(T, \omega)$ such that

$$
\sup _{n} \max _{n \leq k<m(n, T)}\left|\sum_{i=n}^{k} a_{i}\left\langle X_{i}(\omega), Z_{i}\left(X_{i}(\omega), \omega\right)\right\rangle\right| \leq W_{0}(T, \omega), \omega \in \Omega_{0} .
$$

Define $S_{n}^{k}(x)=\sum_{i=n}^{k} a_{i} \delta_{i}^{-2} Z_{i}(x), k \geq n$ and $W(n, k)=\sum_{i=n+1}^{k} a_{i}\left\langle X_{i}, Z_{i}\right\rangle, k \geq n+1$.

Since $Z_{i}(x, \omega) \equiv Z_{i}(x)=a_{i}^{-1} \delta_{i}^{2}\left\{S_{n}^{i}(x)-S_{n}^{i-1}(x)\right\}, i \geq n+1, W(n, k)$ can be rewritten the following formula

$$
W(n, k)=W_{1}(n, k)+W_{2}(n, k)+W_{3}(n, k), k \geq n+1,
$$

where

$$
\begin{aligned}
& W_{1}(n, k)=\sum_{i=n+1}^{k}\left\langle X_{i}, \delta_{i}^{2} S_{n}^{i}\left(X_{i}\right)-\delta_{i-1}^{2} S_{n}^{i-1}\left(X_{i-1}\right)\right\rangle \\
& W_{2}(n, k)=\sum_{i=n+1}^{k}\left\langle X_{i}, \delta_{i-1}^{2}\left\{S_{n}^{i-1}\left(X_{i-1}\right)-S_{n}^{i-1}\left(X_{i}\right)\right\}\right\rangle
\end{aligned}
$$

and

$$
\left.W_{3}(n, k)=\sum_{i=n+1}^{k}\left\langle X_{i},\left(\delta_{i-1}^{2}-\delta_{i}^{2}\right) S_{n}^{i-1}\left(X_{i-1}\right)\right\}\right\rangle
$$

Then we have

$$
\begin{aligned}
& \left|W_{1}(n, k)\right| \leq\left|\sum_{i=n}^{k-1}\left\langle X_{i}-X_{i+1}, \delta_{i}^{2} S_{n}^{i}\left(X_{i}\right)\right\rangle\right|+\left|\left\langle X_{n+1}, \delta_{n}^{2} S_{n}^{n}\left(X_{n}\right)\right\rangle\right| \\
& \quad+\left|\left\langle X_{k}, \delta_{k}^{2} S_{n}^{k}\left(X_{k}\right)\right\rangle\right|, k \geq n+1 .
\end{aligned}
$$

Since $X_{i+1}=X_{i}-a_{i} Y_{i}$, it follows from B0, B5(i), (5.5) and B6 that there exists a positive random variable $\bar{W}_{1}(T, \omega)$ such that

$$
\begin{aligned}
\max _{n \leq k<m(n, T)}\left|W_{1}(n, k)\right| & \leq K \max _{n \leq k<m(n, T)} \sum_{i=n}^{k} a_{i} \delta_{i} \alpha_{i}\left\|S_{n}^{i}\left(X_{i}\right)\right\| \\
& \leq \bar{W}_{1}(T) \quad \text { on } \Omega_{0} .
\end{aligned}
$$

Since $\sup _{n} \delta_{n-1} \delta_{n}^{-1}<x$, it follows from (5.5), B0(iii) and B5(ii) that there exists a positive random variable $\bar{W}_{2}(T, \omega)$ such that

$$
\max _{n \leq k<m(n, T)}\left|W_{2}(n, k)\right| \leq \bar{W}_{2}(T) \quad \text { on } \Omega_{0} .
$$

And we also obtain from B0 and B5(i) 


$$
\max _{n \leq k<m(n, T)}\left|W_{3}(n, k)\right| \leq \bar{W}_{3}(T) \quad \text { on } \Omega_{0} .
$$

Where $\bar{W}_{3}(T)=\bar{W}_{3}(T, \omega)$ is a positive random variable.

Hence (5.11) has been proved. Noting B1 and B4(ii) and applying Lemma 1 to (5.9) it follows from (5.10) and (5.11) that

$$
\sup _{n}\left\|X_{n}\right\|<\infty \quad \text { on } \Omega_{0} .
$$

It is easily seen from B5(i), (ii) that there exists sequence of positive random variables $\left\{\beta_{n}(\omega)\right\}$ such that

$$
\begin{gathered}
\lim _{n \rightarrow \infty} \beta_{n}(\omega)=0 \quad, \omega \in \Omega_{0}, \\
\max _{n \leq k<m(n, T)}\left\|\sum_{i=n}^{k} a_{i} Z_{i}(x, \omega)\right\| \leq \beta_{n}(\omega)(\|x\|+1),(x, \omega) \in H \times \Omega_{0} . \\
\max _{n \leq k<m(n, T)}\left\|\sum_{i=n}^{k} a_{i}\left\{Z_{i}(x, \omega)-Z_{i}(y, \omega)\right\}\right\| \leq \beta_{n}(\omega)\|x-y\|,(x, \omega),(y, \omega) \in H \times \Omega_{0} .
\end{gathered}
$$

Hence using (5.12) to (5.15) and by the similar arguments of the proof of (5.11) we can show that

$$
\lim _{n \rightarrow \infty} \max _{n \leq k<m(n, T)}\left|\sum_{i=n}^{k} a_{i}\left\langle X_{i}, Z_{i}\right\rangle\right|=0 \quad \text { a.s. . }
$$

Hence, by (5.12) and (5.16) and using Lemma 2 we obtain (5.1).

Q.E.D.

REMARK. Let us consider the following assumption.

B5: For any $T>0$ there exists a sequence of positive random variables $\left\{\beta_{n}(\omega)\right\}$ such that (5.13) to (5.15) hold.

And suppose that the conditions of Theorem B hold but with $\sup _{n}\left\|X_{n}(\omega)\right\|<x$, $\omega \in \Omega_{0}$ and $\mathrm{B}^{\prime} 5$ replacing $\mathrm{B} 0$ and $\mathrm{B} 5$, respectively. Then (5.1) also holds.

\section{References}

[1] Borodin, A.N.: A stochastic approximation procedure in the case of weakly dependent observations, Theory Prob. Appl., 24 (1979), 34-52.

[2] FRITZ, J.: Learning from an ergodic training sequence, In "Limit Theorems of Probability Theory"; ed. P. Révész, North-Holland 1974, 79-91.

[3] GyörfI, L.:- Stochastic approximation from ergodic sample for linear regression, Z. Wahrscheinlichkeits theorie verw. Geb. 54 (1980), 47-55.

[4] Kushner, H.J. and Clark, D.S.: Stochastic approximation methods for constrained and unconstrained systems, Springer, 1978.

[5] LJUng, L.: Analysis of stochastic gradient algorithms for linear regression problem, IEEE Information Theory, IT-30 (1984), 151-160.

[6] LuUng, L.: Analysis of recursive stochastic algorithms, IEEE Trans. Automat. Contr., AC-22 (1977), $551-575$. 
[7] Robbins. H. and Monro, S.: A stochastic approximation method, Ann. Math. Statist., 22 (1951), 400407.

[8] Wathane, M.: A stochastic approximation from dependent observations. Z. Wahrsheinlichkeitstheoric verw. Geb., 62 (1983), 279-292.

[9] WatanaBe. M.: The 2rth mean convergence of a stochastic approximation algorithm with weakly dependent observations. Fukuoka Univ. Science Rep., 16 (1986), 71-80.

Received November 28, 1990

Communicated by N. Furukawa 